\title{
External fixation of olecranon fracture associated with soft tissue damage: a case report
}

\author{
Hyong Jun Jon, Tae Song So, Ho Nam Pak \\ Department of Trauma and Orthopaedic Surgery, Pyongyang Medical College, Kim Il Sung University Pyongyang, Democratic People's Republic of \\ Korea \\ Correspondence to: Tae Song So. Department of Trauma and Orthopaedic Surgery, Pyongyang Medical College, Kim Il Sung University Pyongyang, \\ Democratic People’s Republic of Korea. Email: pmed6@ryongnamsan.edu.kp.
}

\begin{abstract}
Olecranon fracture has been operated widely with tension band wiring introduced by Weber and Vasey, or, in case of comminution, with plating. Soft tissue injury around elbow, however, contraindicates the open reduction and internal fixation of olecranon. This study discusses the efficacy of external fixation in case of olecranon fracture associated with soft tissue injury, which has not been well studied yet. Twenty patients (20 elbows) with closed or open olecranon fracture (AO/ASIF classification IC 2-3 \& IO 2) had been treated with olecranon external fixator, and then they were investigated with follow-up period of at least 2 years. Operation time and wound healing problems were recorded, and then final range of motion of the elbow and functional outcome (MEPI) were assessed at the time of the latest follow-up. In this study, the average operation time was $21.5 \pm 5.8 \mathrm{~min}$. There was no deep infection, but only one mild stiffness happened. There was no nonunion. At the time of the latest follow-up, flexion arc of the elbow was $110 \pm 5.0$ degree, and the average MEPI was 90 . This result suggests that severe soft tissue injury associated olecranon fracture which is usually impossible to apply ORIF and that produces a lot of intractable problems can be treated successfully with olecranon external fixator.
\end{abstract}

Keywords: Olecranon; elbow; external fixator; open fracture; soft tissue injury

Received: 22 April 2020; Accepted: 30 December 2020; Published: 30 April 2021.

doi: $10.21037 /$ ht-20-19

View this article at: http://dx.doi.org/10.21037/ht-20-19

\section{Introduction}

Olecranon fracture, which may be the result of indirect or direct trauma, accounts for $10 \%$ of all upper extremity lesions. The treatment of olecranon fracture is almost always operation. The tension band principle introduced by Weber and Vasey has been widely applied, and, in order to avoid migration of the Kirschner wires, intramedullary devices or plate and screw also have been promoted (1-4).

Until now, however, to our knowledge, application of external fixator peculiar to the olecranon fracture has not been reported although other types of external fixators such as hinged external fixator have been described $(5,6)$.

Although most cases of olecranon fracture have been managed with ORIF (open reduction and internal fixation), considerable soft tissue injury around elbow is the contraindication of ORIF. Special external fixator suitable to olecranon stabilization can be the resolution of such cases. The purpose of this study was to investigate the result of olecranon external fixation in the olecranon fracture associated with severe soft tissue injury.

\section{Case presentation}

20 patients with closed or open olecranon fracture (AO/ ASIF classification IC 2-3 \& IO 2) had been treated with olecranon external fixator which we devised in the Department of Trauma and Orthopaedic Surgery, Pyongyang Medical College Affiliated Hospital from Jun. 2008 to Dec. 2015. The patient characteristics are shown in Table 1.

Operation was performed under $\mathrm{X}$-ray $\mathrm{C}$-arm on the 
day of admission, for an average of $21.5 \pm 5.8 \mathrm{~min}$ (range $15-45 \mathrm{~min})$.

Usually within $24 \mathrm{~h}$ after application of external fixator, early motion of the injured elbow was encouraged, and the soft tissue injury was managed simultaneously. At

Table 1 Demographic data of patients

\begin{tabular}{lc}
\hline Distribution & Total $(\mathrm{n}=20)$ \\
\hline AO classification of soft tissue injury & 12 \\
Closed & 8 \\
IC2 & 4 \\
IC3 & 8 \\
Open & 8 \\
IO2 & \\
Gender & 11 \\
Female & 9 \\
Male & \\
Age, year & \\
$20-29$ & 2 \\
$30-39$ & 4 \\
$40-49$ & 6 \\
$50-59$ & 5 \\
$60-69$ & 2 \\
$\geq 70$ & 1 \\
\hline
\end{tabular}

one month after operation, all patients except for one performed more active exercises. It took an average of 2.5 months (range 2-3.5 months) after operation to recover the functional range of elbow motion (over 100 degree of elbow flexion arc). Only one patient had stiffness of the elbow because of wound healing problem and pain. At 2 year postoperatively, ROM of the elbow was $110 \pm 5.0$ degree excluding one elbow stiffness.

All patients experienced pin track infection, but it was resolved immediately after removal of external fixation pins, which were removed at 6-8 weeks when fracture union was confirmed. Deep infection was not developed.

Soft tissue damage did not compromise the process of bony union so that all fracture sites were united successfully. All patients were followed up for at least 2 years. Outcome assessment was done with MEPI (Mayo Elbow Performance Index) at the latest visit. At the latest followup, the MEPI score was 90 of 100 scores on average.

\section{Illustrative case (see Figure 1)}

A 42-year-old man sustained his right olecranon fracture (Schatzker type2, AO/ASIF classification IC3) due to traffic road accident. On the day of admission, olecranon external fixator was applied and then patient was encouraged to use his injured elbow by rotating and flexing and extending on the day after operation. After that, pin sites were managed everyday during the period of external fixator application. At 7 weeks postoperatively union of fracture site was confirmed so that the external device was removed. At the
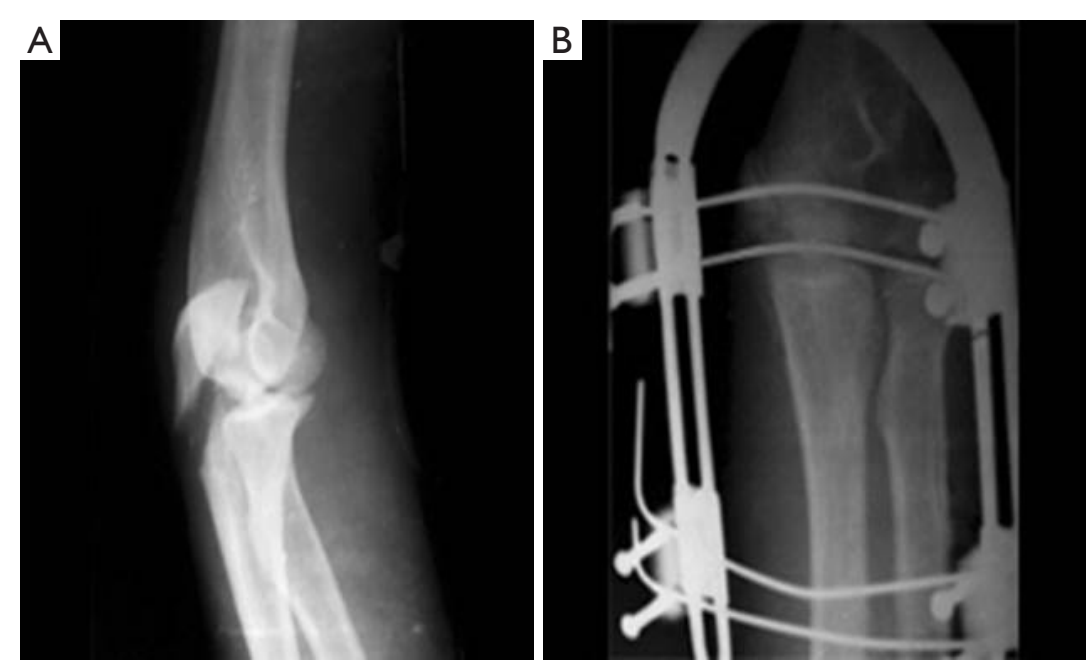

Figure $1 \mathrm{X}$-ray finding of an illustrative case. (A) Preoperative X-ray finding; (B) postoperative X-ray finding. 
last visit, final ROM of his right elbow was 120 degree and the MEPI score was 100 . The patient was satisfactory with the result of treatment.

All procedures performed in studies involving human participants were in accordance with the ethical standards of the institutional and national research committee and with the Helsinki Declaration (as revised in 2013) and its later amendments or comparable ethical standards. Informed consent was obtained from all individual participants included in the study.

\section{Discussion}

Until now, olecranon fracture has been treated by tension band wiring, intramedullary nailing or plate fixation $(1-3,4)$.

Application of external fixator peculiar to the olecranon fracture, however, has not been reported although other types of external fixators such as hinged external fixator have been described (6).

External fixator has been widely applied in other parts of extremities due to lots of advantages, and has become an essential part of damage control surgery in polytrauma and one option for the skeletal stabilization of open fractures, particularly in those with severe soft-tissue injury as it permits rapid stabilization of fractures with minimal additional (surgical) injury. It allows "local damage control" for fractures with severe soft-tissue injuries (5).

Thus, in case of open olecranon fracture or closed olecranon fracture associated with extensively contused soft tissue around elbow which delays or contraindicates the ORIF so that compromises the recovery of the injured elbow, external fixation should be applied.

We performed external fixation in 20 patients with softtissue injury associated olecranon fracture in order to not only recover the elbow function as early as possible but also simultaneously manage the wound and we report the result of treatment here in this investigation.

As shown in Table 1, we applied this new type of olecranon external fixator only to the cases associated with severe soft-tissue damage such as AO/ASIF classification IC 2-3, IO 2, which are the contraindication of early open reduction and internal fixation. All patients included in this investigation were operated on the day of admission with olecranon external fixator under image intensifier, and then their wounds were managed everyday. At postoperative 6-8 weeks all cases showed osseous union of fracture site. At 2 years, the elbows of all cases were clinically stable, and no further deformity or stiffness has occurred except for only one case. An extension deficit of 10-30 degree remained in all cases.

The mean Mayo Elbow Performance Index was 90 of 100 points at the latest follow-up.

Though the present data shows the promising aspect of olecranon external fixator in the management of olecranon fracture with soft tissue injury, the current study has some limitations.

The number of sample was modest. This study included just only 20 patients (20 elbows). In addition, this study just subjected only AO/ASIF classification IC 2-3, IO 2, which could be one of the causes of bias. We can not be sure what happens if we extends the application of this technique to more severe type of soft tissue damaged fracture. Especially in case of flap plasty and external fixation, it might be impossible to begin early mobilization of the injured elbow immediately after operation. If the study includes all cases treated with this current technique further, the result might be some different, revealing other important factors affecting outcomes. Furthermore, this hardware is cumbersome so that it might affect the patients` satisfaction level because patients feel uncomfortable during application of this external device, which has not been considered in this study.

In conclusion, olecranon fracture with severe soft tissue damage can be successfully managed with olecranon external fixator, although it is cumbersome to apply the hardware around the injured elbow, but further investigation should be performed to exactly clarify the efficacy and shortcomings of external fixation in the management of olecranon fracture.

\section{Acknowledgments}

Funding: None.

\section{Footnote}

Conflicts of Interest: All authors have completed the ICMJE uniform disclosure form (available at http://dx.doi. org/10.21037/ht-20-19). The authors have no conflicts of interest to declare.

Ethical Statement: The authors are accountable for all aspects of the work in ensuring that questions related to the accuracy or integrity of any part of the work are appropriately investigated and resolved. All procedures performed in studies involving human participants were in accordance with the ethical standards of the institutional and national research committee and with the Helsinki 
Declaration (as revised in 2013) and its later amendments or comparable ethical standards. Informed consent was obtained from all individual participants included in the study.

Open Access Statement: This is an Open Access article distributed in accordance with the Creative Commons Attribution-NonCommercial-NoDerivs 4.0 International License (CC BY-NC-ND 4.0), which permits the noncommercial replication and distribution of the article with the strict proviso that no changes or edits are made and the original work is properly cited (including links to both the formal publication through the relevant DOI and the license). See: https://creativecommons.org/licenses/by-nc-nd/4.0/.

\section{References}

1. Joshi RP. The Hastings experience of the Attenborough

doi: $10.21037 / \mathrm{ht}-20-19$

Cite this article as: Jon HJ, So TS, Pak HN. External fixation of olecranon fracture associated with soft tissue damage: a case report. Health Technol 2021;5:6. springs and Rush nail for fixation of olecranon fractures. Injury 1997;28:455-8.

2. Nowinski RJ, Nork SE, Segina DN, et al. Comminuted fracture-dislocations of the elbow treated with an $\mathrm{AO}$ wrist fusion plate. Clin Orthop Relat Res 2000;(378):238-44.

3. Rommens PM, Küchle R, Schneider RU, et al. Olecranon fractures in adults: factors influencing outcome. Injury 2004;35:1149-57.

4. Weber BG, Vasey H. Osteosynthesis in olecranon fractures. Z Unfallmed Berufskr 1963;56:90-6.

5. Rüedi TP, Buckley R, Moran CG. AO Principles of Fracture Management. Ann R Coll Surg Engl 2009;91:448-9.

6. Volkov MV, Oganesian OV. Restoration of function in the knee and elbow with a hinge-distractor apparatus. J Bone Joint Surg Am 1975;57:591-600. 